\title{
AVALIAÇÃO DE RISCO FÍSICO: EXPOSIÇÃO OCUPACIONAL AOS NÍVEIS DE PRESSÃO SONORA EM UMA INDÚSTRIA DE TRANSFORMAÇÃO
}

\author{
PHYSICAL RISK ASSESSMENT: OCCUPATIONAL EXPOSURE OF SOUND \\ PRESSURE LEVELS IN THE MANUFACTURING INDUSTRY
}

\author{
Danilo Simões, Gislaine Cristina Batistela \\ Universidade Estadual Paulista "Júlio de Mesquita Filho"- UNESP, Câmpus de \\ Itapeva, Itapeva, SP. \\ E-mail: danilo.simoes@unesp.br
}

\begin{abstract}
RESUMO - O ruído pode ser considerado como uma das principais causas de doenças ocupacionais dentre as formas de energia caracterizadas como riscos físicos. Objetivou-se avaliar a exposição dos operadores de máquinas de uma indústria de transformação aos níveis de pressão sonora para comparação com os níveis estabelecidos pela legislação brasileira e determinação da dose diária que os operadores estão expostos. $O$ estudo foi desenvolvido em uma serraria que realiza o desdobro de madeira, localizada no Estado de São Paulo. Os níveis de ruído foram coletados durante toda a jornada diária de trabalho por meio de um dosímetro de uso pessoal. Os resultados evidenciaram que dentre os postos de trabalho ponderados no estudo, somente um posto não possui nível de ruído acima do estabelecido pela legislação vigente. Logo, torna-se imperativo a utilização de equipamentos de proteção individual e de equipamentos de proteção coletiva, ademais, recomenda-se o enclausuramento acústico das máquinas geradoras de ruído acima de 115 dBA. Palavras-chave: ergonomia; higiene e segurança; posto de trabalho.
\end{abstract}

Recebido em: 20/09/2017

Revisado em: 27/02/2018

Aprovado em: 28/02/2018

\begin{abstract}
The noise can be considered as one of the leading causes of occupational diseases among the forms of energy characterized as physical risks. This work aimed at evaluating of sound pressure levels that workers of an manufacturing industry are exposure for compare with the levels established by Brazilian legislation and determine the daily dose that workers are exposed to. The study was developed in a sawmill that performs the wood split, located in São Paulo State. The noise levels were collected throughout the daily work, by means of a personal dosimeter. The results showed that among the job positions analyzed in this study, only one station doesn't have the sound pressure levels above the levels established by current legislation. Therefore, the use of personal and collective protective equipment's is needful, moreover, it is recommended the acoustic enclosure of noise-generating machines above $115 \mathrm{dBA}$.
\end{abstract}

Keywords: ergonomics; hygiene and safety; workstation. 


\section{INTRODUÇÃO}

Dentre o vasto universo de atividades ocupacionais, encontram-se as realizadas em serrarias e marcenarias (OLIVEIRA et al., 2009). No entendimento de Sobieray et al. (2007) as atividades nas indústrias madeireiras são consideradas atividades de risco, ou seja, sujeita aos mais variados tipos de acidente. Para Souza et al. (2002) essas indústrias de transformação ocupam posição de destaque, pela frequência relativa $e$ gravidade dos acidentes de trabalho, sobretudo, pelo tipo de dano causado ao trabalhador, frequência de lesões permanentes, óbitos e pelo longo período de afastamento do trabalho.

As máquinas e ferramentas utilizadas nesses locais propiciam a realização de atividades com sobrecargas físicas e riscos biomecânicos (FIEDLER; VENTUROLI, 2002; FIEDLER et al., 2008). Esse ambiente ocupacional expõe os trabalhadores aos riscos, que podem ser periculosos ou insalubres. Os agentes periculosos são àqueles mais visíveis que podem afetar a integridade física do trabalhador, enquanto que os agentes insalubres são mais insidiosos, atuando em longo prazo, minando paulatinamente a saúde do trabalhador (OLIVEIRA, 2002).

Portanto, o trabalhador de uma indústria do setor madeireiro, segundo Fiedler e Souza (2007) pode estar sujeito a diferentes situações de exposição no ambiente de trabalho, como, exemplo, elevados níveis de ruído. Para Arezes e Miguel (2012) este agente físico constitui um dos riscos ocupacionais com maior expressão na indústria transformadora.

O ruído é definido por Ganime et al. (2010) como som ou grupo de sons com intensidade que pode culminar em adoecimentos ou interferências negativas no processo de comunicação. Para Filipe et al. (2014) o ruído é o nome habitual atribuído à pressão sonora, em que o som se caracteriza por flutuações de pressão em um meio compressível, como o ar, a água, a madeira e outros materiais, não se propagando no vácuo, sendo esse um risco insalubre, presente em quase todo tipo de instalação industrial.

Nessa perspectiva, o ruído indica a magnitude de uma quantidade física, o qual é uma mistura complexa de diversas vibrações, medido na escala logarítmica, cuja unidade é o decibel (dB), que acima do limiar da sensação dolorosa, pode produzir danos ao aparelho auditivo (IIDA, 2005).

Para Moraes (2010) o agente ruído, de modo geral, constitui-se em um dos maiores riscos para a saúde dos trabalhadores, tanto nas instalações industriais como em outras atividades laborais. Este é certamente um dos agentes físicos nocivos mais frequentes no ambiente de trabalho, e de maior prevalência das origens de doenças ocupacionais (CRUZ et al., 2013).

Guida et al. (2010) salientam que o ruído, isoladamente, apresenta perigo à saúde quando o nível sonoro é superior a 85 dBA, dependendo da duração e exposição sistemática a ele e, por esta razão, a partir desta intensidade sonora, faz-se audiometria, periodicamente em indústrias.

Conforme Lopes et al. (2009) em quase todos os processos do beneficiamento da madeira, o ruído está presente e a falta de manutenção e instalações adequadas, aliadas a um layout ineficiente, contribuem para o aumento do ruído nos ambientes das madeireiras, além do desconhecimento e a negligência em relação às práticas seguras, que são evidenciados nessas indústrias, onde se encontram níveis de ruído que variam de 90 a 120 dBA.

Ao considerar que o ruído ocupacional é um risco físico frequente em quase todos os segmentos industriais (ALMEIDA et al., 2000), além de aumentar a ocorrência de acidentes de trabalho (CHIOVENDA et al., 2007), atribui aos trabalhadores acidentes de trabalho, como exemplo, dificuldades de comunicação (na deteç̧ão, discriminação, localização e identificação das fontes sonoras, assim como na inteligibilidade de fala), manutenção da 
atenção e concentração, estresse e fadiga excessiva (CORDEIRO et al., 2005).

Com o objetivo de prevenir o dano auditivo, Akatsu e Sá (2013) esclarecem que - levantamento do nível de ruído e informações encontradas em estudos tornam-se importantes meios de comparação com os limites de tolerância estabelecidos pela legislação brasileira, como, os Anexos 1 e 2 da Norma Regulamentadora 15 (NR 15). Essa Norma, estabelece limites de tolerância para ruídos, que pode ser contínuo ou intermitente, quando não há variação do nível de pressão sonora nem do espectro sonoro; flutuante, quando apresenta variações de nível de energia acústica em função do tempo; ou de impacto, aquele que apresenta picos de energia acústica de duração inferior a um segundo, a intervalos superiores a um segundo (BRASIL, 1978).

Diante desse contexto, em ambientes ocupacionais, que podem gerar níveis de ruído acima do permitido pela legislação em vigor, recomenda-se a avaliação quantitativa do risco físico de ruído ocupacional com vistas à adoção de instrumentos que possam mitigar as doenças ocupacionais, seja por meio do uso de equipamentos de proteção individual ou de proteção coletiva, no entanto, que permitam resguardar a audição dos operadores expostos à níveis de ruído contínuo ou intermitente.

Sob este prisma, o objetivo deste estudo foi avaliar a exposição dos operadores de máquinas empregadas no desdobro de madeira, aos níveis de pressão sonora, para comparação com os níveis estabelecidos pela legislação brasileira e determinação da dose diária que estes operadores estão expostos.

\section{MATERIAL E MÉTODOS}

\subsection{Local do estudo}

O estudo foi desenvolvido em uma serraria localizada no Estado de São Paulo, que realiza o desdobramento de Pinus spp., com toras de diâmetros entre $18 \mathrm{~cm}$ e $25 \mathrm{~cm}$, destinadas à fabricação de produtos de madeira. Ademais, as operações da serraria eram orientadas pelo fluxo contínuo de produção, conforme Rother e Shook (1998).

A jornada de trabalho diária dos operadores era de 8 horas, a qual iniciava-se às 7 horas e encerrava-se às 17 horas, com intervalo de almoço entre 11 horas e 13 horas. Estes operadores possuíam em média 6,2 anos de exposição ocupacional ao ruído.

Para as medições do nível de ruído ocupacional foram ponderados todos os postos de trabalho (etapas de produção) essenciais para a obtenção do produto final, caracterizados de acordo com a máquina, sendo estas: serra destopadeira, serra fita (entrada e saída), serra múltipla, picador de madeira, serra quádrupla e serra refiladora, além do posto de trabalho necessário para a classificação do produto final, que era realizada manualmente, isto é, sem o emprego de máquinas ou equipamentos. Isto posto, a amostra ponderou 8 trabalhadores, ou seja, visou caracterizar a exposição de todos os trabalhadores da serraria, devido contemplar as condições operacionais e ambientais de todos postos de trabalho.

\subsection{Procedimentos de avaliação do ruído ocupacional}

Os limites de exposição ocupacional ao ruído e incremento de duplicação de dose foram pautados na Norma Regulamentadora 15 - NR 15 (BRASIL, 1978), por ser aceita no Brasil pela legislação previdenciária em vigor para fins de insalubridade.

Os dados foram coletados durante 8 dias, isto é, um dia para cada posto de trabalho avaliado durante toda a jornada diária de trabalho, por meio de um dosímetro de uso pessoal da marca Instrutherm modelo DOS-600, embasado no critério de avaliação da exposição diária dos operadores ao ruído contínuo, com critério de referência de 85 dBA, que correspondeu a dose de $100 \%$.

O valor de nível de pressão sonora (Equação 1) expresso em decibels (dB), que de acordo com Saliba (2015) é o que determina a intensidade do som e é expressa pelo logaritmo da relação entre a variação da pressão provocada pela vibração e a pressão 
que atinge o limiar de audibilidade, foi coletado a cada 60 segundos.

$N P S=20 \log \left(\frac{P}{P_{0}}\right)$

em que:

$N P S$ - nível de pressão sonora em decibel

(dB);

$P$ - pressão sonora instantânea em pascal $(\mathrm{Pa})$;

$P_{0}$ - pressão sonora de referência, igual a $20 \times 10^{-6} \mathrm{~Pa}$, em $1000 \mathrm{~Hz}$.

Para a obtenção do nível de ruído equivalente (Leq), que pondera todos os níveis de ruído que o trabalhador experimentou durante o tempo real da jornada de trabalho, empregou-se a Equação 2.

Leq

$=L c+q \log \left[\frac{D(Q) \times T c}{100 \times T}\right]$

em que:

Lc - nível base do critério, igual a $85 \mathrm{dBA}$;

Tc - período do nível base do critério de avaliação, igual a 8 horas;

$T$ - duração das medições, igual a 8 horas;

$D(Q)$ - dose de ruído, em porcentagem;

$q$-incremento de duplicação de dose.

A dose de ruído é caracterizada pelo efeito aditivo de níveis de ruídos específicos que o trabalhador fica exposto durante a jornada diária de trabalho, descrita na Equação 3.

$$
=\sum_{i=1}^{n} \frac{C_{i}}{\operatorname{Tmax}_{i}}
$$

em que:

$C_{i}$ - tempo real diário de exposição a um nível de ruído específico;

$\operatorname{Tmax}_{i}$ - tempo máximo de exposição ao correspondente nível de ruído da norma adotada.

O tempo máximo de exposição diária permissível em função do nível de ruído foi calculado por meio da Equação 4.

$\operatorname{Tmax}=\frac{8}{2^{(N P S-85) / q}}$

Destarte, foi adotada a especificação para dosímetro pessoal de ruído conforme a norma ANSI S1.25 (AMERICAN STANDARDS
INSTITUTE, 1991), isto é, o nível de pressão sonora foi medido em decibéis ( $d B$ ) com o dosímetro no circuito de ponderação " $A$ " $e$ circuito de resposta lenta (slow), dBA, $q=5$, nível limiar de integração 80 dBA e indicação da ocorrência de níveis superiores a 115 dBA.

Portanto, aplicou-se o procedimento técnico para a coleta dos níveis de ruído consonante à Fundacentro (2001). Por fim, o dosímetro foi acoplado à calça dos operadores, com o microfone posicionado e fixado sobre o ombro destes.

Para análise estatística foi calculado o valor médio do nível de ruído a cada 30 minutos, que considerou o tempo total de 480 minutos da jornada diária de trabalho, em cada posto, configurando 16 blocos. Desta forma, ponderou-se um modelo em blocos completos ao acaso, com 8 tratamentos caracterizados pelos postos de trabalho, conforme o modelo da Equação 5.

$y_{i j}=\mu+\tau_{i}+\beta_{j}+\varepsilon_{i j}$

em que:

$y_{i j}$ - observação do NPS referente ao posto de trabalho $i$ no bloco de tempo $j$;

$\mu$ - média geral comum a todas as observações;

$\tau_{i}$ - efeito do $i$-ésimo posto de trabalho, com $i=1,2, \ldots, 8$;

$\beta_{j}$ - efeito do $j$-ésimo bloco de tempo, $j=1$,

$2, \ldots, 16$;

$\varepsilon_{i j}$ - erro aleatório ou resíduo.

Para os contrastes entre as médias do

(3) NPS em cada posto de trabalho, os quais possibilitaram indicar os resultados das comparações entre todos os pares de médias, foi utilizado 0 teste Tukey considerando o nível de 5\% de significância (MONTGOMERY, 2012).

\section{RESULTADOS E DISCUSSÕES}

O nível de pressão sonora é determinante para distinguir as fontes sonoras de um sistema produtivo. Deste modo, o maior nível de pressão sonora foi obtido no posto de trabalho da serra quádrupla, o que também ocorreu em estudo realizado por Venturoli et al. (2003) com uma 
máquina semelhante à analisada. Cabe ressaltar, que o nível de pressão sonora é intrínseco à potência nominal $(k W)$ e à rotação do motor (rpm) da máquina e, ainda, da resistência da madeira.

Ao comparar os níveis de pressão sonora em todos os postos de trabalho (Tabela 1), esse nível diferiu estatisticamente somente no posto de trabalho da serra refiladora, onde foi constatado o menor nível de pressão sonora $(77,7 \mathrm{dBA})$, ou seja, o único posto de trabalho que o nível de pressão sonora não ultrapassou o limite de 85 dBA estabelecido pela NR 15. Não obstante, o nível médio de pressão sonora na serraria foi de $88,9 \mathrm{dBA}$, portanto, acima do limite de tolerância máximo de exposição para uma jornada diária de trabalho de 8 horas, que segundo Kroemer e Grandjean (2005) está próximo ao limiar prejudicial.

Gonçalves et al. (2015) salientam que a exposição ao ruído intenso ocupacional pode ser minimizada com a utilização de equipamentos de proteção auditiva de uso individual, isto é, os protetores auriculares. Fernandes e Morata (2002) destacam que a perda auditiva induzida por ruído (PAIR) são alterações dos limiares auditivos do tipo neurossensorial, decorrentes da exposição ocupacional sistemática a níveis elevados de pressão sonora, e tem como características principais a irreversibilidade e a progressão gradual com o tempo de exposição ao risco. Por conseguinte, para a condição analisada, torna-se imprescindível a utilização de protetores auriculares ou abafadores acústicos pelos operadores posicionados nesses postos de trabalhos, isto é, adoção de medidas que visam minimizar doenças ocupacionais, como exemplo, a perda da audição.

Em relação ao tempo máximo de exposição diária de trabalho permissível para o operador de acordo com a NR-15, no posto de trabalho na serra quádrupla sem protetores auriculares será de aproximadamente 2 horas, sendo que o único posto de trabalho analisado que não demanda proteção auricular para uma jornada diária de trabalho de 8 horas foi no posto da serra refiladora. Então, a partir do nível médio de pressão sonora emitido na serraria é possível $6 \mathrm{~h}$ e $11 \mathrm{~min}$ de trabalho diário sem protetores auriculares. No entanto, Rodrigues et al. (2006) salientam que há uma diferença no valor da atenuação do ruído entre os protetores auriculares e essa diferença pode aumentar dependendo da colocação do protetor. Por consequência disso, torna-se fundamental o esclarecimento aos operadores para o uso adequado desses protetores.

Tabela 1. Nível médio de pressão sonora emitido nos postos de trabalho da serraria e tempo máximo de exposição diária permitida.

\begin{tabular}{lll}
\hline Posto de trabalho & NPS (dBA) & $\begin{array}{l}\text { Tempo máximo de } \\
\text { exposição }\end{array}$ \\
\hline Classificação & $88,7 \mathrm{a}^{*}$ & $4 \mathrm{~h} 47 \mathrm{~min}$ \\
Serra destopadeira & $87,2 \mathrm{a}$ & $5 \mathrm{~h} 54 \mathrm{~min}$ \\
Serra fita (entrada) & $89,0 \mathrm{a}$ & $4 \mathrm{~h} \mathrm{36min}$ \\
Serra fita (saída) & $94,1 \mathrm{a}$ & $2 \mathrm{~h} 16 \mathrm{~min}$ \\
Serra múltipla & $89,7 \mathrm{a}$ & $4 \mathrm{~h} \mathrm{10min}$ \\
Picador de madeira & $90,0 \mathrm{a}$ & $3 \mathrm{~h} \mathrm{32 \textrm {min }}$ \\
Serra quádrupla & $94,5 \mathrm{a}$ & $2 \mathrm{~h} 9 \mathrm{~min}$ \\
Serra refiladora & $77,7 \mathrm{~b}$ & $8 \mathrm{~h}$ \\
\hline
\end{tabular}

\footnotetext{
* médias seguidas de letras iguais não diferem estatisticamente entre si $(p>0,05)$ pelo teste de Tukey
}

Para Oliveira et al. (2011) o Leq é uma informação importante para análise dos resultados, uma vez que o mesmo possibilita conhecer o nível sonoro médio integrado, 
durante um determinado período de tempo, sabendo-se que lesões à audição humana são provocadas não só por exposição a ruídos elevados, mas também, pela duração dos mesmos, portanto, de acordo com Lopes et al. (2004) é a medida mais correta, pois registra não somente os diferentes níveis de ruído encontrados, mas também o seu tempo de duração.

Diante disso, o operador que desempenhava atividades junto à serra quádrupla foi o que mais estava exposto ao dano auditivo, que fundamentado no Leq (Tabela 2) desse posto de trabalho e no tempo médio de exposição ocupacional dos operadores ao NPS (6,2 anos), tem $12 \%$ de chance de perda auditiva conforme a NP1733 (PORTUGAL, 1981). É importante salientar que a adição de $5 \mathrm{~dB}$, ou seja, o incremento de duplicação de dose empregado no estudo, pode duplicar o risco de lesão da capacidade auditiva do operador. A partir desses resultados, justifica-se a adoção de medidas que visam à proteção da saúde dos trabalhadores, que pode ser assentada em um Programa de Conservação Auditiva (PCA).

De acordo com Rios (2007) a implantação de um PCA torna-se necessário em locais de trabalho onde os níveis de exposição ao ruído estejam acima dos limites de tolerância. Oliveira et al. (2012) salientam que a função principal do PCA é prevenir a evolução das perdas auditivas decorrentes da exposição ao ruído ocupacional.

Tabela 2. Nível de ruído equivalente real nos postos de trabalho da serraria.

\begin{tabular}{ll}
\hline Posto de trabalho & Leq (dBA) \\
\hline Classificação & 92,9 \\
Serra destopadeira & 99,7 \\
Serra fita (entrada) & 93,7 \\
Serra fita (saída) & 96,3 \\
Serra múltipla & 98,3 \\
Picador de madeira & 96,9 \\
Serra quádrupla & 101,3 \\
Serra refiladora & 96,9 \\
\hline
\end{tabular}

De acordo com Sales et al. (2015) quando ocorrem dois ou mais períodos de exposição a ruído de diferentes níveis, a maneira correta de se avaliar o ruído é por meio da dose diária. À vista disso, observa-se na Tabela 3 que a dose diária de ruído extrapolou o critério de referência para o limite de exposição diária (100\%) em todos os postos de trabalho, consequentemente, o ambiente de trabalho da serraria pode ser considerado insalubre. Dentre os postos de trabalhos avaliados, os três postos que podem propiciar mais patologias ocupacionais aos operadores durante a execução das tarefas, são a serra quádrupla, serra destopadeira e o picador de madeira, os quais expõem os trabalhadores às doses aproximadas de $650 \%, 540 \%$ e $420 \%$, respectivamente.

Deste modo, estes percentuais estão em média $537 \%$ superior ao permissível para uma jornada de trabalho de 8 horas. Como consequência, segundo Oliveira et al. (2015) irão gerar impactos negativos à saúde auditiva do trabalhador como o desenvolvimento da PAIR, assim como implicações na qualidade de vida e aumento do estresse. 
Tabela 3. Valor da dose de ruído recebido pelos operadores nos postos de trabalho da serraria.

\begin{tabular}{ll}
\hline Posto de trabalho & Dose (\%) \\
\hline Classificação & 126,3 \\
Serra destopadeira & 643,4 \\
Serra fita (entrada) & 128,0 \\
Serra fita (saída) & 190,6 \\
Serra múltipla & 247,1 \\
Picador de madeira & 521,7 \\
Serra quádrupla & 753,3 \\
Serra refiladora & 191,8 \\
\hline
\end{tabular}

\section{CONSIDERAÇÕES FINAIS}

Em consonância à Norma Regulamentadora 15, o tempo máximo diário da jornada de trabalho permissível na serraria analisada sem o correto uso de protetores auriculares deverá ser reduzida em 2 horas e 49 minutos.

O nível de ruído ocupacional médio foi $4,6 \%$ acima do valor estipulado pela Norma Regulamentadora 15 para uma jornada de trabalho de 8 horas.

o valor médio do nível de ruído equivalente que o trabalhador recebeu foi de 97 dBA.

As máquinas que geraram níveis de pressão sonora acima do valor teto de 115 dBA, devem ter enclausuramento acústico.

Os níveis elevados de pressão sonora além da prevalência de alterações auditivas, podem provir demais doenças que impactam no absenteísmo.

\section{REFERÊNCIAS}

ALMEIDA, S. I. C. et al. História natural da perda auditiva ocupacional provocada por ruído. Revista da Associação Médica Brasileira, São Paulo, v. 46, n. 2, p. 143-158, 2000. Disponível em <http://www.scielo.br/pdf/ramb/v46n2/2842.pdf >. Acesso em: 15 jun. 2017.

AMERICAN STANDARDS INSTITUTE. ANSI S1.25: specifications for personal noise dosimeters. Washington, 1991.

AKATSU, S. Y.; SÁ, E. C. Avaliação pericial do ruído em oficina de locomotivas situada na cidade de São Paulo. Saúde, Ética \& Justiça, v. 18, n. 1, p. 124-127, 2013. Disponível em
$<$ https://www.revistas.usp.br/sej/article/view/75 144/78702>. Acesso em: 12 jun. 2017.

AREZES, P. M.; MIGUEL, A. S. A exposição ocupacional ao ruído em Portugal. Revista Portuguesa de Saúde Pública. v. 20, n. 1. p. 61-69, $2002 . \quad$ Disponível em: $<$ https://www.ensp.unl.pt/dispositivos-deapoio/cdi/cdi/sector-depublicacoes/revista/2000-2008/pdfs/1-062002.pdf>. Acesso em: 16 jun. 2017.

BRASIL. Ministério do Trabalho e Emprego. NR-15 Atividades e Operações Insalubres. Brasília, 1978. Disponível em $<$ http://trabalho.gov.br/images/Documentos/SST LNR/NR15/NR15-ANEXO15.pdf>. Acesso em: 12 jun. 2017.

CHIOVENDA, P. et al. Environmental noiseexposed workers: Event--related potentials, neuropsychological and mood assessment. International Journal of Psychophysiology. v. 65, n. 3, p. 228-237, 2007. Disponível em <https://doi.org/10.1016/j.ijpsycho.2007.04.009> . Acesso em: 18 jun. 2017.

CORDEIRO, R. et al. Exposição ao ruído ocupacional como fator de risco para acidentes do trabalho. Saúde Pública. v. 39, n. 3, p. 461-66, $2005 . \quad$ Disponível em $<$ http://dx.doi.org/10.1590/S003489102005000300018>. Acesso em: 16 jun. 2017.

CRUZ, F.; LAGO, E. M. G.; BARBOKÉBAS JUNIOR, $B$. Evaluation of noise generated by propagation equipment beat stakes construction site. In: AREZES, P. M.; BAPTISTA, J. S.; BARROSO, M. P.; CARNEIRO, P.; CORDEIRO, P. COSTA, N.; MELO, R. B.; MIGUEL, A. S.; PERESTRELO, G. Ocupacional 
safety and hygiene. London: CRC Press, 2013. https://doi.org/10.1201/b14391-90

FERNANDES, M.; MORATA, T. C. Estudo dos efeitos auditivos e extra-auditivos da exposição ocupacional a ruído e vibração. Revista Brasileira de Otorrinolaringologia, v. 68, n. 5, p. 705-713, $2002 . \quad$ Disponível em $<$ http://dx.doi.org/10.1590/S0034-

72992002000500017>. Acesso em: 18 jun. 2017.

FIEDLER, N. C. et al. Análise da carga física de trabalho dos operadores em marcenarias no sul do Espírito Santo. Floresta, v. 38, n. 3, p.413-419, $2008 . \quad$ Disponível em: $<$ http://revistas.ufpr.br/floresta/article/view/124 07/8530>. Acesso em: 14 mai. 2017.

FIEDLER, N. C.; SOUZA, A. P. Ergonomia e segurança do trabalho na indústria madeireira. In: TECNOLOGIAS APLICADAS AO SETOR MADEIREIRO, 2. Anais... Vitória: Aquarius, 2007. p. 217-244.

FIEDLER, N. C.; VENTUROLI, F. Avaliação da carga física de trabalho exigida em atividades de fabricação de móveis no Distrito Federal. Cerne, v. 8 n. 2, p. 117-122, 2002. Disponível em: $<$ http://www.cerne.ufla.br/site/index.php/CERNE Larticle/view/589>. Acesso em: 09 jun. 2017.

FILIPE, A. P. et al. Avaliação de ruído em fábricas de móveis. Cerne, v. 20, n. 4, p. 551-556, 2014. Disponível em: <http://dx.doi.org/10.1590/01047760201420049 50>. Acesso em: 12 mai. 2017.

FUNDACENTRO. Norma de Higiene Ocupacional 01: procedimento técnico para a avaliação da exposição ocupacional ao ruído contínuo ou intermitente e impacto. São Paulo. 2001.

GANIME, J. F. et al. O Ruído como um dos riscos ocupacionais: uma revisão de literatura. Enfermería Global, v. 19, n. 2, p. 1-15, 2010. Disponível em: $<$ http://revistas.um.es/eglobal/article/view/1073 21/102711>. Acesso em: 14 mai. 2017.

GONÇALVES, C. G. O. et al. A percepção sobre protetores auriculares por trabalhadores participantes de programas de preservação auditiva: estudo preliminar. CoDAS, v. 27, n. 4, p. 309-318, 2015. Disponível em:
$<$ http://dx.doi.org/10.1590/23171782/20152014139>. Acesso em: 22 mai. 2017.

KROEMER, K. H. E.; GRANDJEAN. E. Manual de ergonomia: adaptando o trabalho ao homem. 5. ed. Porto Alegre: Bookman, 2005.

GUIDA, H. L.; MORINI, R. G.; CARDOSO, A. C. V. Audiological evaluation in workers exposed to noise and pesticide. Brazilian Journal of Otorhinolaryngology, v. 76, n. 4, p. 423-427, $2010 . \quad$ Disponível em: $<$ http://dx.doi.org/10.1590/S1808$86942010000400003>$. Acesso em: 16 mai. 2017.

IIDA, I. Ergonomia: projeto e produção. São Paulo: Edgard Blücher, 2005.

LOPES, E. S. et al. Análise do ambiente de trabalho em indústrias de processamento de madeira na região Centro-Sul do Estado do Paraná. Scientia Forestalis, v. 66, n. 2, p. 183-190, $2004 . \quad$ Disponível em: $<$ http://www.ipef.br/publicacoes/scientia/nr66/c ap18.pdf>. Acesso em: 18 set. 2017.

LOPES, A. C. et al. Alterações auditivas em trabalhadores de indústrias madeireiras do interior de Rondônia. Revista Brasileira de Saúde Ocupacional, v. 34, n. 119, p. 88-92, 2009. Disponível em: <http://dx.doi.org/10.1590/S0303-

$76572009000100010>$. Acesso em: 12 mai. 2017.

MONTGOMERY, D. C. Design and analysis of experiments. 8. ed. New York: John Wiley \& Sons, 2012.

MORAES, M. V. G. Doenças Ocupacionais agentes: físico, químicos, biológico, ergonômico. São Paulo: Érica, 2010.

OLIVEIRA, A. G. S.; BAKKE, H. A.; ALENCAR, J. F. Riscos biomecânicos posturais em trabalhadores de uma serraria. Fisioterapia e Pesquisa, v. 16, n. 1 , p. 28-33, 2009. Disponível em: <http://www.scielo.br/pdf/fp/v16n1/06.pdf>. Acesso em: 24 jul. 2017.

OLIVEIRA, F. L. et al. Nível de ruído em sala de parto. Texto contexto-Enfermagem, v. 20, n. 2, p. 287-293, 2011. Disponível em: <http://www.scielo.br/pdf/tce/v20n2/a10v20n2. pdf>. Acesso em: 17 ago. 2017. 
OLIVEIRA, R. C. et al. Ruído Ocupacional e tripulantes da ambulância. Revista CEFAC, v. 17, n. 3, p. 847-853, 2015. Disponível em: <http://www.scielo.br/pdf/rcefac/v17n3/19820216-rcefac-17-03-00847.pdf>. Acesso em: 08 jun. 2017.

OLIVEIRA, S. G. Proteção jurídica à saúde do trabalhador. São Paulo: LTr, 2002.

OLIVEIRA, W. T. G. H. et al. Audição de trabalhadores antes e após o programa de conservação auditiva. Revista Brasileira de Ciências da Saúde. v. 16, n. 4. p. 517-524, 2012. Disponível em: <http://www.okara.ufpb.br/ojs/index.php/rbcs/a rticle/view/12411>. Acesso em: 16 set. 2017.

PORTUGAL. Instituto Português da Qualidade. NP-1733: Estimativa da exposição ao ruído durante o exercício de uma actividade profissional com vista à protecção da audição. Lisboa: Instituto Português da Qualidade, 1981.

RIOS, A. L. Implantação de um programa de conservação auditiva: enfoque fonoaudiológico. 2007. Tese (Doutorado) - Faculdade de Medicina de Ribeirão Preto da Universidade de São Paulo, $2007 . \quad$ Disponível em: <http://www.teses.usp.br/teses/disponiveis/17/1 7138/tde-13112007-150213/pt-br.php>. Acesso em: 16 set. 2017.

RODRIGUES, M. A. G.; DEZAN; A. A.; MARCHIORI, L. L. M. Eficácia da escolha do protetor auricular pequeno, médio e grande em programa de conservação auditiva. CEFAC. v. 8, n. 4, p. 543547, 2006. Disponível em: <http://dx.doi.org/10.1590/S1516-

18462006000400016>. Acesso em: 14 set. 2017.

ROTHER, M.; SHOOK, J. Learning to see: value stream mapping to add value and eliminate muda. Cambridge: The Lean Enterprise Institute, 1998.

SALES, R.; SILVA, F. M.; SILVA, F. C. Doses de ruído a qual estão submetidos operadores de derriçadoras portáteis de café. Coffee Science, Lavras, v. 10, n. 2, p. 169-175. 2015. Disponível em:

<http://www.coffeescience.ufla.br/index.php/Co ffCoffeesci/article/view/808/pdf 169>. Acesso em: 04 set. 2017.
SOBIERAY, T. N. C. et al. Um estudo sobre o uso de equipamentos de proteção como prevenção de acidentes em indústrias madeireiras de Mato Grosso. Revista Eletrônica do Mestrado em Educação Ambiental, v. 18, n. 1, p. 268-282, 2007. Disponível em: <https://www.seer.furg.br/remea/article/view/3 553>. Acesso em: 13 set. 2017.

SOUZA, V.; BLANK, V.; CALVO, M. Cenários típicos de lesões decorrentes de acidentes de trabalho na indústria madeireira. Revista Saúde Pública, v. 36, n. 6, p. 702-708, 2002. Disponível em: <http://dx.doi.org/10.1590/S0034-

89102002000700007>. Acesso em: 12 jun. 2017.

VENTUROLI, F. et al. Avaliação do nível de ruído em marcenarias no Distrito Federal, Brasil. Revista Brasileira de Engenharia Agrícola e Ambiental, v. 7, n. 3, p. 547-551, 2003. Disponível em:

<http://www.scielo.br/pdf/rbeaa/v7n3/v7n3a23. pdf>. Acesso em: 30 ago. 2017. 